\title{
Dual-energy X-ray absorptiometry and fracture prediction in patients with spinal cord injuries and disorders: methodological issues
}

\author{
S. Sabour ${ }^{1,2}$ iD
}

Received: 17 February 2017 / Accepted: 23 February 2017 /Published online: 13 May 2017

(C) International Osteoporosis Foundation and National Osteoporosis Foundation 2017

\section{Dear Editor,}

I was interested to read the paper by Abderhalden and colleagues published in the March 2017 issue of Osteoporosis International [1]. Persons with a spinal cord injury (SCI) have substantial morbidity and mortality following osteoporotic fractures. The aim of the authors was to determine whether dual-energy X-ray absorptiometry (DXA) measurements predict osteoporotic fractures in this population. Additionally, the authors suggested that osteopenia $(\mathrm{OR}=4.7)$ or osteoporosis $(\mathrm{OR}=4.3)$ compared with normal BMD was significantly associated with fractures and higher $T$ scores at the hip were inversely associated with fractures $(\mathrm{OR}=0.7)$. There was no significant association of $T$ scores or World Health Organization (WHO) classification with incident fractures in those with complete SCI ( $p>0.15$ for both). The study suggested that DXA-derived measurements at the hip predict fracture risk in persons with a SCI [1].

However, this result has nothing to do with prediction. First, significant association does not necessarily mean prediction. Moreover, for prediction studies, we need data from two different cohorts or at least from one cohort divided into two to first to develop a prediction model and subsequently validate it. Misleading results are generally the main outcome of research that fails to validate its prediction models [2-7].

A response to these comments is available at doi 10.1007/s00198-0174056-6.

S. Sabour

s.sabour@sbmu.ac.ir

1 Safety Promotion and Injury Prevention Research Center, Shahid Beheshti University of Medical Sciences, Tehran, Iran

2 Department of Clinical Epidemiology, School of Health, Shahid Beheshti University of Medical Sciences, Tehran, Iran
Finally, in prediction studies, we must assess the interactions between important variables. Final results can be impacted dramatically when qualitative interactions are present [2-7]. This means that most of the time, without assessing the interaction terms, prediction studies will mainly produce misleading messages.

\section{Compliance with ethical standards}

Conflict of interest No disclosures.

Permissions Not relevant.

\section{References}

1. Abderhalden L, Weaver FM, Bethel M, Demirtas H, Burns S et al (2017) Dual-energy X-ray absorptiometry and fracture prediction in patients with spinal cord injuries and disorders. Osteoporos Int 28(3): 925-934. doi:10.1007/s00198-016-3841-y

2. Rothman KJ, Greenland S, Lash TL (2008) Cohort studies. In: Rothman KJ (ed) Modern Epidemiology, 3rd edn. Lippincott Williams \& Wilkins, Baltimore, pp 79-85

3. Sabour S (2015) Letter by Sabour regarding article, "Coated-platelets improve prediction of stroke and transient ischemic attack in asymptomatic internal carotid artery stenosis". Stroke 46(2):e46

4. Sabour S (2015) Prediction of preterm delivery using levels of VEGF and leptin in amniotic fluid from the second trimester: prediction rules. Arch Gynecol Obstet 291(4):719

5. Sabour S (2015) Prediction of 3-dimensional pharyngeal airway changes after orthognathic surgery: a methodological issue. Am J Orthod Dentofac Orthop 147(1):8

6. Sabour S (2014) Obesity predictors in people with chronic spinal cord injury: a common mistake. J Res Med Sci 19(1):80

7. Sabour S, Ghassemi F (2013) Predictive value of confocal scanning laser for the onset of visual field loss. Ophthalmology 120(6):e31-e32 